\title{
Temporal Response of MSM-LED Optical Integrated Device
}

\author{
Sh. M. Eladl and M. A. Ashour \\ Radiation Engineering Department, NCRRT, Atomic Energy Authority, 3 Ahmed Alzomor St., 8th Sector, \\ P.O. Box 29, Nasr City, Cairo, Egypt \\ Correspondence should be addressed to Sh. M. Eladl,shaban_45@yahoo.com
}

Received 20 October 2010; Accepted 20 January 2011

Academic Editor: Ali Hussain Reshak

Copyright ( $) 2011$ Sh. M. Eladl and M. A. Ashour. This is an open access article distributed under the Creative Commons Attribution License, which permits unrestricted use, distribution, and reproduction in any medium, provided the original work is properly cited.

\begin{abstract}
The temporal response of an optical integrated device is theoretically analysed. The device is composed of a Metal-SemiconductorMetal (MSM) Photodetector and a Light Emitting Diode (LED). The analysis is based on the frequency response of the constituent devices without any optical feedback within the device structure. All expressions describing the frequency response, time response, output derivatives, and rise time are outlined. The results show that the temporal response of MSM detectors is similar to the LED response if the cutoff frequency of the LED is lower than that of MSM, while it is similar to MSM detector if the cutoff frequency of the MSM is lower than that of LED.
\end{abstract}

\section{Introduction}

There is an increased interest in Metal-Semiconductor-Metal (MSM) photodetectors recent years as they offer an attractive alternative to the traditional choice of PIN photodetectors. The MSM Photodetectors are very suitable for high-speed photodetection. This increased interest is because of their ease of fabrications, larger bandwidth, and flexibility with other electronic circuit technologies. Monolithic integration of such types of devices onto several semiconducting materials allows them to be integrated with other semiconducting devices to obtain other enhanced functions $[1,2]$.

Planar metal-semiconductor-metal light-emitting diodes with InGaN/GaN multiquantum wells (MQWs) as the active layer have been demonstrated for the first time. The diodes with interdigitated Schottky electrodes fabricated on p-GaN contact layer exhibit symmetrical current-voltage characteristic with a turn-on voltage of $\sim 13 \mathrm{~V}$ at $20 \mathrm{~mA}$. The violet light emission centred at $408 \mathrm{~nm}$ is generated by radiative recombination taking place in the MQWs. The evolution of light output power against injection current reveals an enhanced carrier collecting efficiency of the active MQWs at higher injection current level $[3,4]$.

The reliable $\mathrm{n}^{+}-\mathrm{ZnSSe}$ metal-semiconductor-metal (MSM) blue-green light emitting diodes (LEDs) have been fabricated. The contact metal was $\mathrm{CuGe/Pt} / \mathrm{Au}$. The current transport mechanisms agree very well with the back to back tunneling diodes. The kink phenomena were observed in the MSM current-voltage curves. In the metal-semiconductor interface, the element $\mathrm{Zn}$ in $\mathrm{ZnSSe}$ can be replaced by $\mathrm{Cu}$ results in some acceptor levels as radiative recombination centers in the MS interface. The peak wavelength in the LED electroluminescent (EL) spectra was strongly dependent on the injection currents from 5 to $40 \mathrm{~mA}$. The peak wavelength and full width at half maximum are 510 and $10 \mathrm{~nm}$, respectively, at $10 \mathrm{~mA}$ injection current. When the injection current increases to $15 \mathrm{~mA}$, the peak wavelength shifted to $530 \mathrm{~nm}$ due to different recombination centers. Further increasing the injection currents, the peak wavelength shifted slightly to the long wavelength side [5-7]. No previous attention has been focused to analyze the MSM-LED integrated device.

In this paper, a proposed integrated optical device is composed of a Metal-Semiconductor-Metal (MSM) photodetector as a detecting part at the input side of the device and a Light Emitting Diode (LED) as an emitting part at the output side of the device. A schematic view of the proposed structure is shown in Figure 1; the device consists of MSM detector with interdigitated contacts each separated by a distance $L$. The other part of the device is 3 layers 


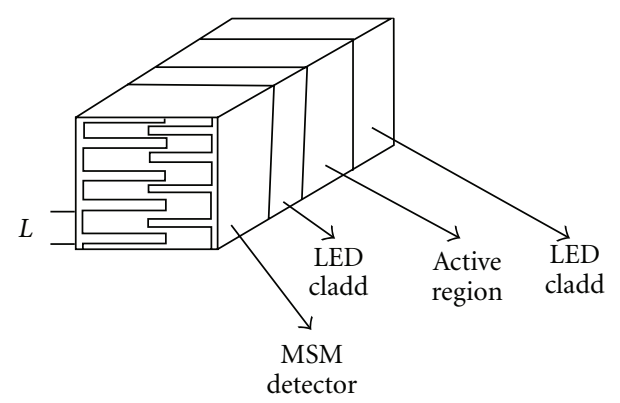

FIGURE 1: A schematic view of MSM-LED integrated device.

of LED. The aim is to study the dynamic characteristics of this integrated device based on the frequency response concerning this two constituent parts. An optical feedback will take place due to the back light from the LED side to the MSM photodetector side within the structure of the device. The analytical expressions are carried out by neglecting this optical feedback effect within the device due to the difference in the optical spectra of both input and output sides.

\section{Theoretical Analysis}

The frequency response of the quantum efficiency of MSM photodetector is outlined in [8]

$$
\eta_{\mathrm{MSM}}(\omega)=\frac{h v}{\tau_{t r}} \sum_{k=1}^{\infty} \frac{C_{k, n}}{\zeta_{k, n}+j \omega},
$$

where $h$ is blank's constant, $v$ is the frequency of the input light, and $C_{k, n}, \zeta_{k, n}, \tau_{t r}$ are described in [8].

And the frequency response of LED is reduced as

$$
\eta_{\mathrm{LED}}(\omega)=\frac{\eta_{0}}{1+j \omega / \omega_{\mathrm{LED}}} .
$$

For MSM-LED integrated structure,

$$
\eta_{\mathrm{MSM}-\mathrm{LED}}(\omega)=\left(\frac{h \nu}{\tau_{t r, n}} \sum_{k=1}^{\infty} \frac{C_{k, n}}{\zeta_{k, n}+j \omega}\right)\left(\frac{\eta_{0}}{1+j \omega / \omega_{\mathrm{LED}}}\right) .
$$

Assuming a unit impulse response of the input light power

$$
\eta_{\mathrm{MSM}-\mathrm{LED}}(s)=\frac{h \nu}{\tau_{t r, n}}\left(\frac{1}{s}\right)\left(\sum_{k=1}^{\infty} \frac{C_{k, n}}{\zeta_{k, n}+s}\right)\left(\frac{\eta_{0}}{1+s / \omega_{\mathrm{LED}}}\right) .
$$

Taking the inverse Laplace,

for $\omega_{\mathrm{LED}}>\varsigma_{k, n}$,

$$
\eta_{\mathrm{MSM}-\mathrm{LED}}(t)=\frac{h \nu C_{k, n}}{\tau_{t r, n} \zeta_{k, n}}\left[1-\sum_{k=1}^{\infty} \eta_{0} e^{-\varsigma k, n t}\right],
$$

for $\omega_{\mathrm{LED}}<\varsigma_{k, n}$,

$$
\eta_{\mathrm{MSM}-\mathrm{LED}}(t)=\frac{C_{k, n}}{\zeta_{k, n}}\left[1+\eta_{0} e^{-\omega_{\mathrm{LED}} t}\right] .
$$

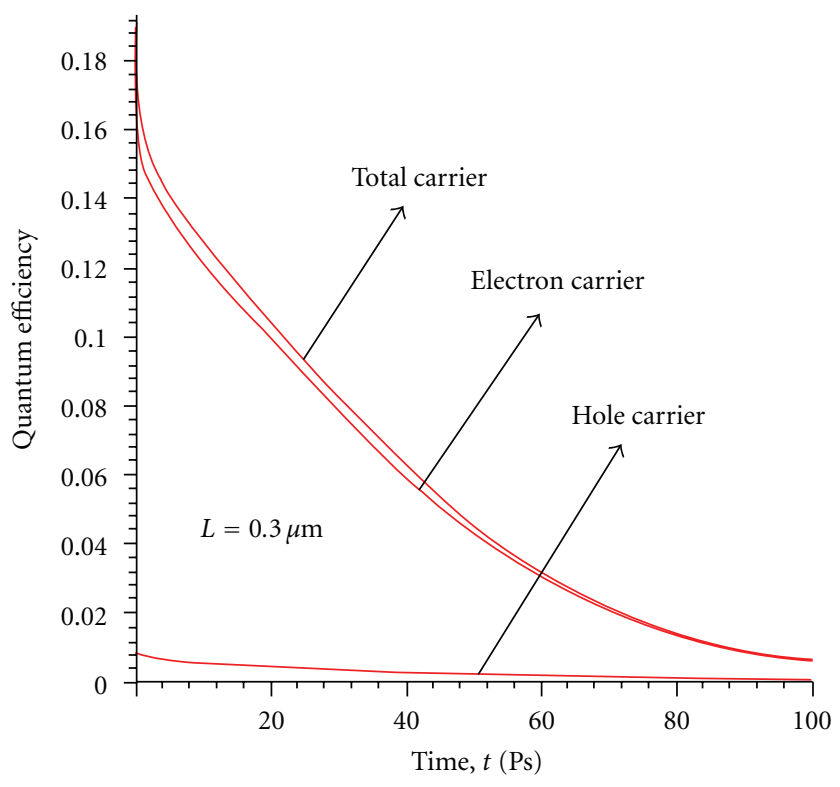

FIgURE 2: Temporal response of MSM at $L=0.3 \mu \mathrm{m}$.

The output derivative of MSM-LED as a measure of speed can be derived as

$$
\frac{d}{d t}\left(\eta_{\text {MSM-LED }}(t)\right)=\frac{h \nu\left[C_{k, n}\right]^{2}}{\tau_{t r, n} \zeta_{k, n}}\left[\sum_{k=1}^{\infty} \eta_{0} e^{-s k, n t}\right] .
$$

And for $\omega_{\mathrm{LED}}<\varsigma_{k, n}$, it will reduce as

$$
\frac{d}{d t}\left(\eta_{\mathrm{MSM}-\mathrm{LED}}(t)\right)=\frac{-\omega_{\mathrm{LED}} C_{k, n}}{\zeta_{k, n}}\left[\eta_{0} e^{-\omega_{\mathrm{LED}} t}\right] .
$$

Rise time can be calculated as follows:

$$
T=\ln \left[\frac{0.2\left[h \nu C_{k, n}\right]-0.9 \eta \zeta_{k, n} \tau_{t r, n} / \eta_{0}}{\left[h \nu\left(C_{k, n}\right)^{2}\right]}\right]^{-1} .
$$

\section{Results and Discussions}

The device parameters used in the following calculations are as follows: $\tau=1 \mathrm{~ns}, k=20, \mu_{n}=8500 \mathrm{~cm}^{2} / \mathrm{V}$.s, and $\mu_{p}=$ $400 \mathrm{~cm}^{2} /$ V.s. The input light is assumed as a step function in time. The transient response of the quantum efficiency of MSM photodetector for carriers of electrons, holes, and total photocurrents is shown in Figure 2. This curve is calculated by 20 terms of the Fourier series concerning (1). The steeply sloped curve is for the electron and the much less sloped curve, appearing almost as constant for this current scale, is for the holes. The total photocurrent appears approximately as the electron current offset by a constant. In this regime, it is evident because of their higher mobility. The dependence of quantum efficiency on the contact spacing $L$ is clear in Figure 3. It can be seen that the high quantum efficiency of the device can be achieved by choosing the contact spacing $L$ to be large. The calculated transient response of the MSM-LED integrated device is shown in 


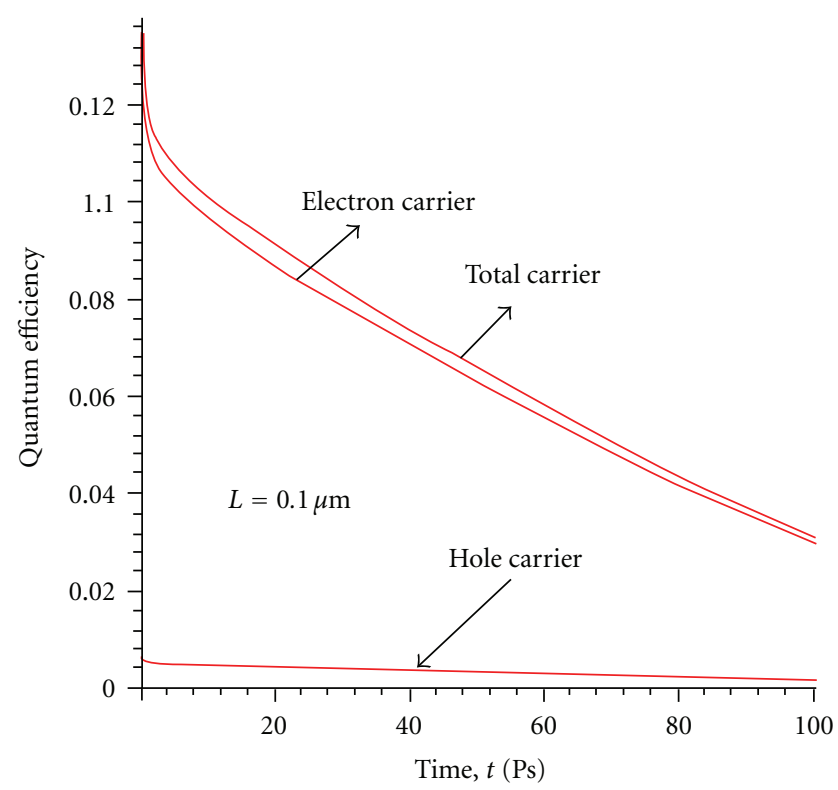

FIgURE 3: Temporal response of MSM at $L=0.1 \mu \mathrm{m}$.

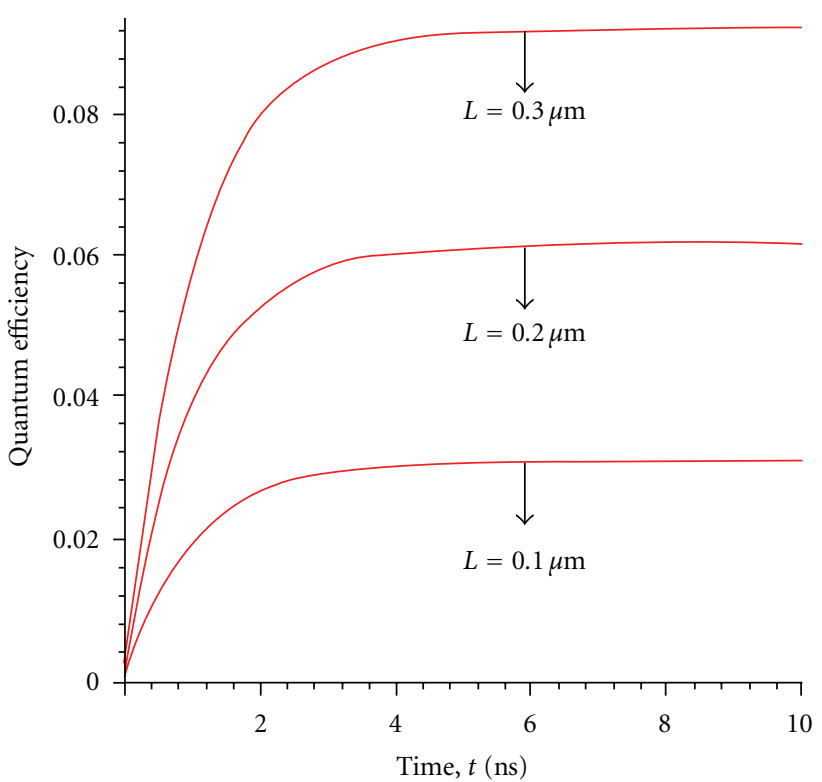

FIGURE 4: Temporal response of MSM-LED at different values of $L$ and $\mu=8500 \mathrm{vcm}^{-2}$.

Figure 4. It can be noticed that the contact spacing $L$ has a major effect on controlling the quantum efficiency of the device. Also shown in the figure as the contact spacing $L$ is increased, which in turn increases the width of the barrier, the quantum efficiency of the considered device is also increased. In addition, the quantum efficiency value initially increases linearly with time until approaching a definite value; this definite value mainly depends on the input power intensity, operating wavelength, and the contact spacing $L$. The parameters used for calculation are shown in the figures.

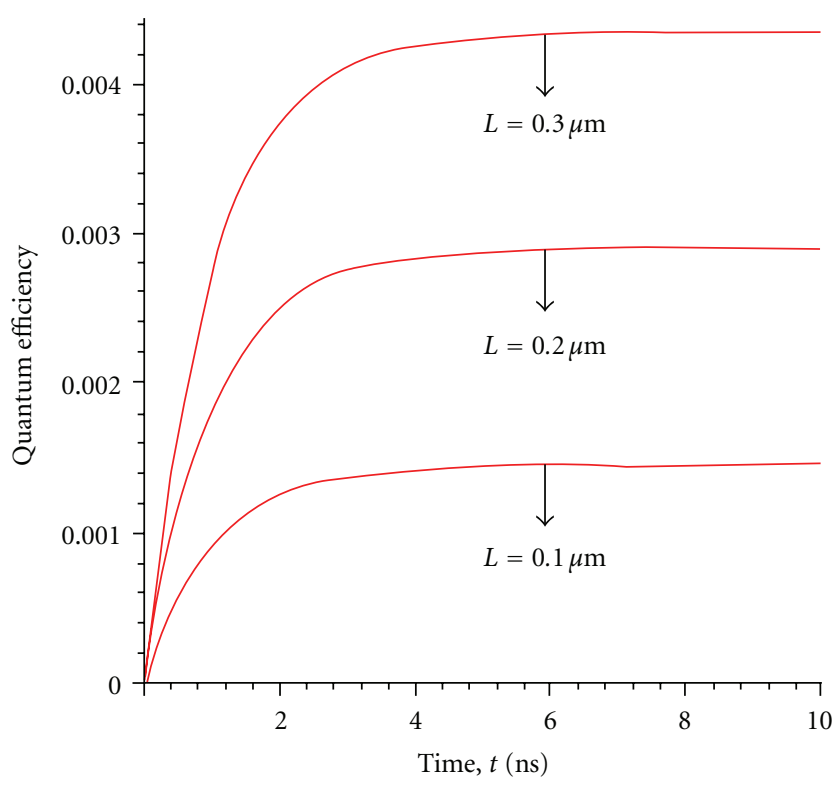

FIgURE 5: Temporal Response of MSM-LED at different values of $L$ and $\mu=400 \mathrm{vcm}^{-2}$.

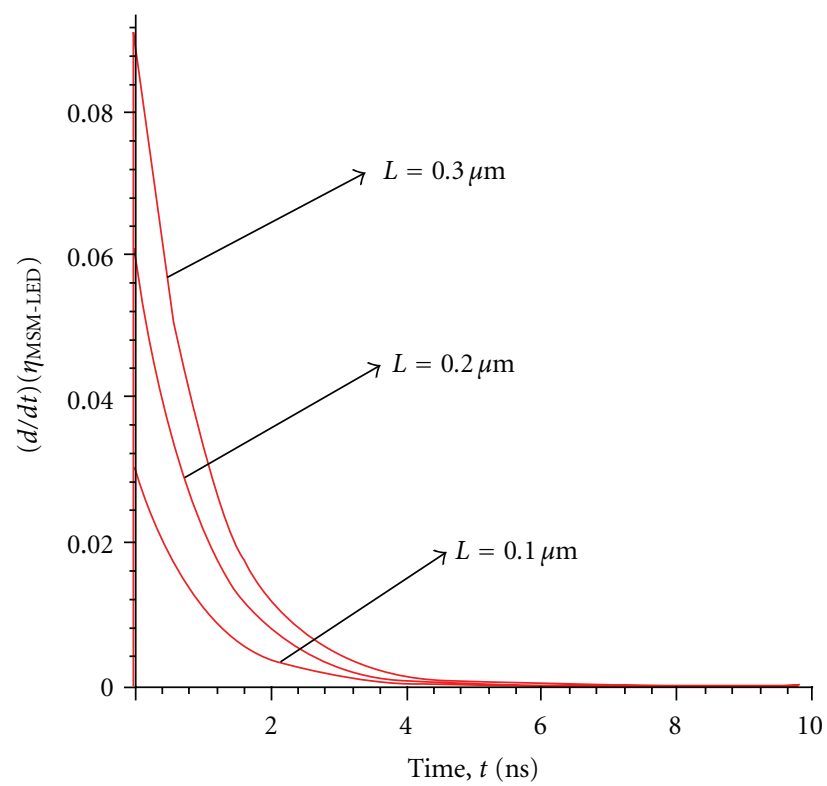

FIgURE 6: Output derivative of MSM-LED at different values of $L$ and $\mu=8500 \mathrm{vcm}^{-2}$.

The value of carrier mobility $\mu$ has a significant effect on the performance evaluation of the MSM-LED integrated device. Figure 5 shows the temporal response of the quantum efficiency of the developed device when carrier mobility $\mu$ is taken into account. From the figure, as the value of carrier mobility $\mu$ increases the quantum efficiency of the proposed device decreases.

The dependence of the output derivative as a measure of the device speed on time is shown in Figure 6. Also the effect of contact spacing $L$ on the output derivative is shown in the same figure. It has been shown from the figure that 


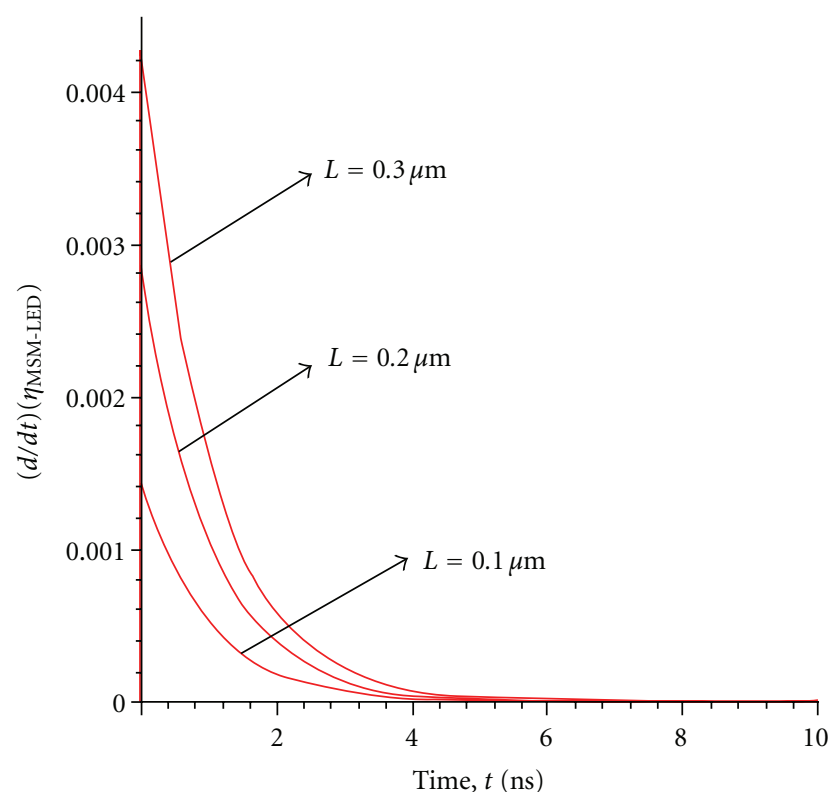

Figure 7: Temporal Response of MSM-LED at different values of $L$ and $\mu=400 \mathrm{vcm}^{-2}$.

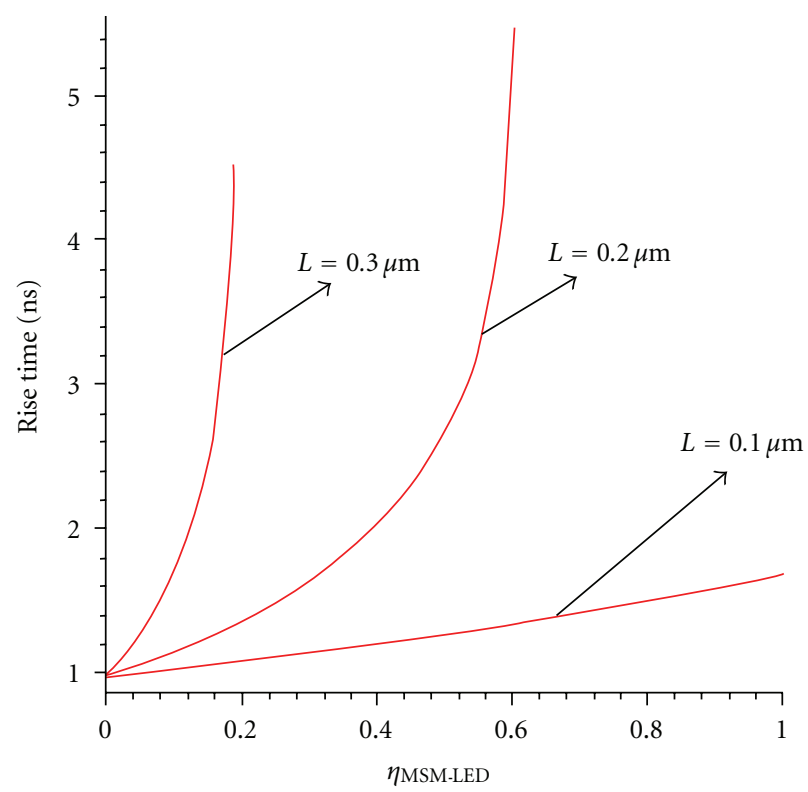

FIGURE 8: Rise time against quantum efficiency of MSM-LED at different values of $L$.

the device output changes with decreasing derivative and thus will approach zero, also the output derivative increases with the increase of contact spacing $L$ at any time.

Again, the value of carrier mobility $\mu$ has a significant effect on the output derivative of the MSM-LED integrated device. Figure 7 shows the variation of the output derivative of the considered model with time when $\mu$ is taken into account to be considered. From the figure, as the value of carrier mobility $\mu$ increases the device output changes with decreasing derivative.
The variation of the rise time with the desired quantum efficiency at different values of $L$ is shown in Figure 8. It can be noticed that the rise time increases with the increase of quantum efficiency and the contact spacing $L$ of the device. With the increase in the desired quantum efficiency, the difference between the final state quantum efficiency and the initial one will increase; this increased difference will take more time and hence the increase in rise time. Also, the quantum efficiencies of both MSM photodetector and LED cause an enhancement of the output, and hence the rise time is increased as a result.

\section{Conclusion}

Analytical modeling of the temporal performance of an integrated device is presented. The device under study is composed of a Metal-Semiconductor-Metal (MSM) Photodetector and a Light Emitting Diode (LED). The modeling is based on the frequency response of the constituent devices. The analytical expressions describing the transient performance are derived. The results show that the quantum efficiency of LED causes an enhancement of the output and rise time is increased as a result. As a future extension to this study, modeling of this device by the use of its equivalent circuit is planned to show the effect of all interesting parameters on the static characteristics

\section{References}

[1] C. Miao, H. Lu, X. Z. Du, Y. Li, R. Zhang, and Y. D. Zheng, "InGaN/GaN multi-quantum-well planar metalsemiconductor-metal light-emitting diodes," Electronics Letters, vol. 44, no. 6, pp. 441-443, 2008.

[2] Y. K. Su, W. R. Chen, S. J. Chang et al., "The red shift of ZnSSe metal-semiconductor-metal light emittingdiodes with high injection currents," IEEE Transactions on Electron Devices, vol. 47, no. 7, pp. 1330-1333, 2000.

[3] X. Chen, The research and fabrication of GaN-based materials and MSM UV-photodetectors, M.S. thesis, Xiamen University, 2002.

[4] L. Liu, Study of ohmic contact and MSM UV detector based on GaN, M.S. thesis, Zhejiang University, 2004.

[5] J. Zhang, Y. Yang, Y. Lu, L. Lou, and Y. Zhao, "Simulation and analysis of $4 \mathrm{H}-\mathrm{SiC}$ metal-semiconductor-metal ultraviolet photodetector," Chinese Journal of Lasers, vol. 35, no. 4, pp. 509514, 2008 (Chinese).

[6] M. Mosca, J. L. Reverchon, N. Grandjean, and J. Y. Duboz, "Multilayer (Al,Ga)N structures for solar-blind detection," IEEE Journal on Selected Topics in Quantum Electronics, vol. 10, no. 4, pp. 752-758, 2004.

[7] Z. Wu, X. Xin, F. Yan, and J. Zhao, "Fabrication of MSM structure UV photodetector on 4H-SiC," Chinese Journal of Quantum Electronics, vol. 21, p. 269, 2004, in Chinese.

[8] A. W. Sarto and B. J. Van Zeghbroeck, "Photocurrents in a metal-semiconductor-metal photodetector," IEEE Journal of Quantum Electronics, vol. 33, no. 12, pp. 2188-2194, 1997. 

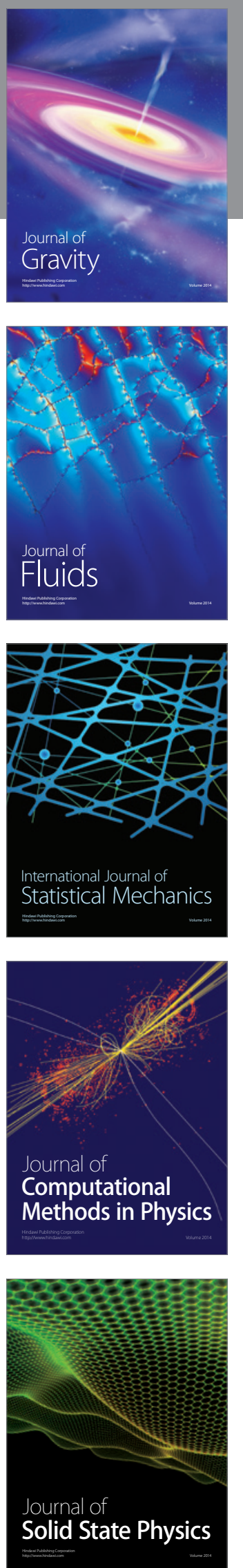

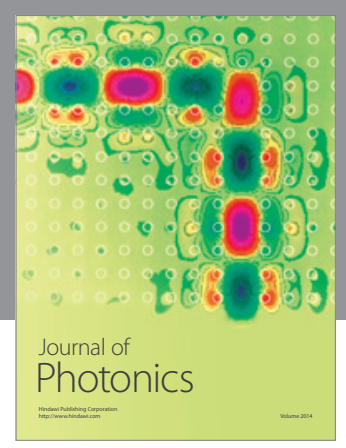

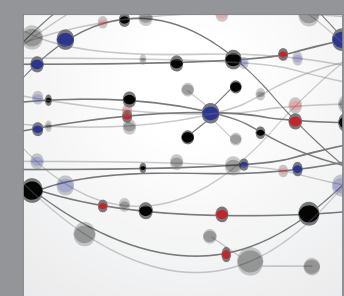

The Scientific World Journal
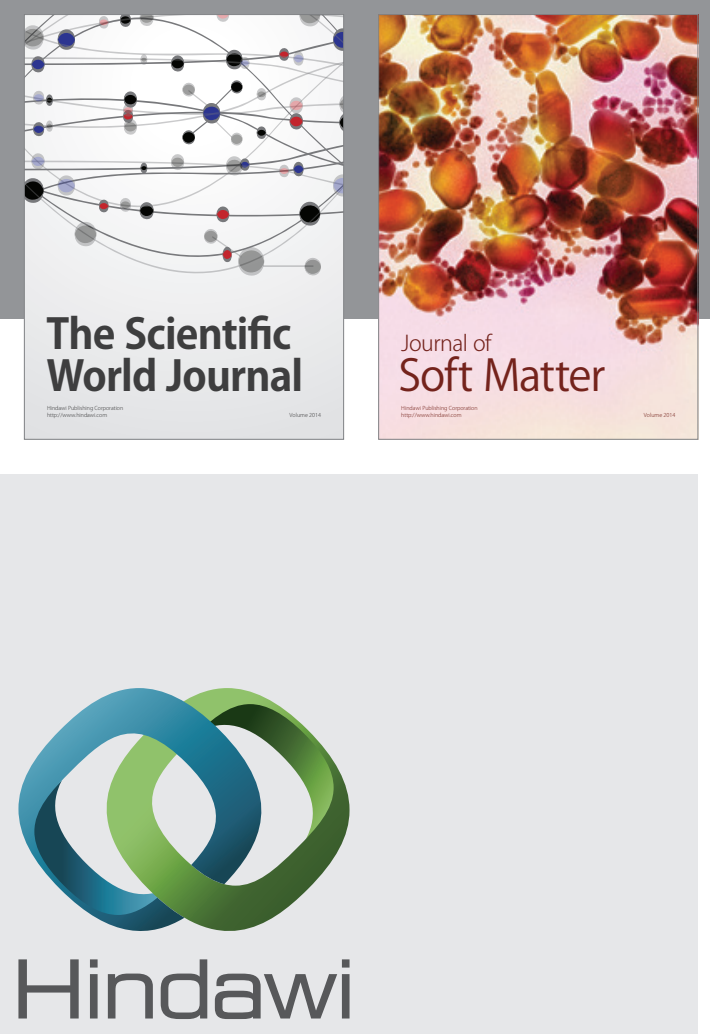

Submit your manuscripts at

http://www.hindawi.com
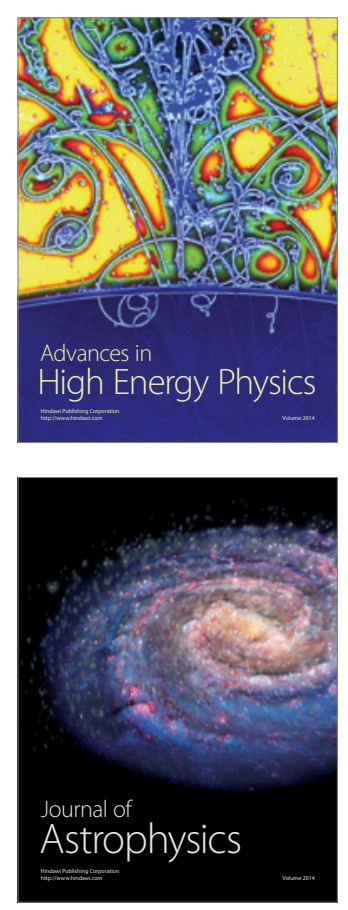
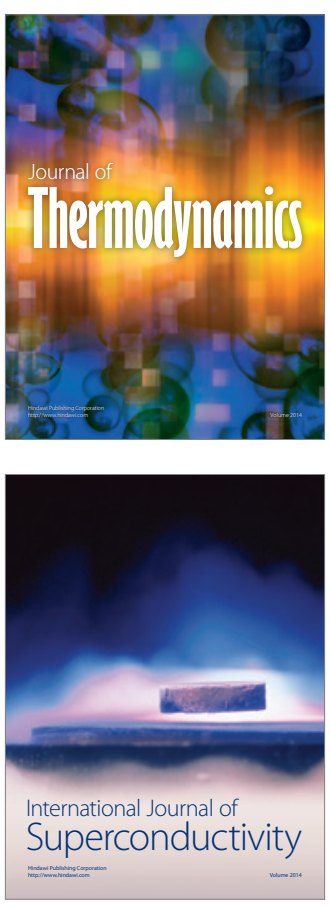
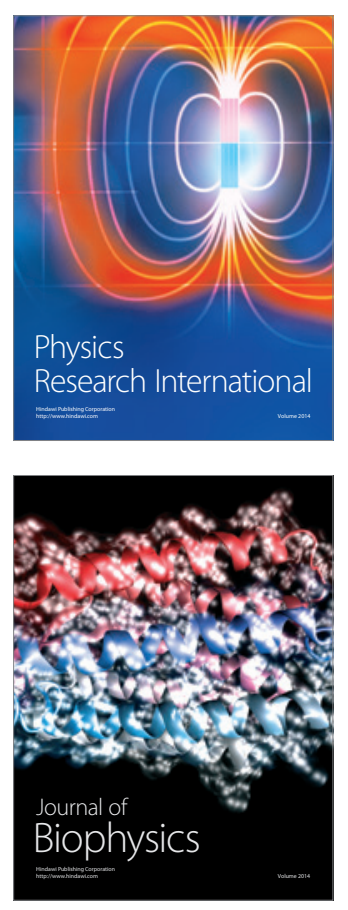
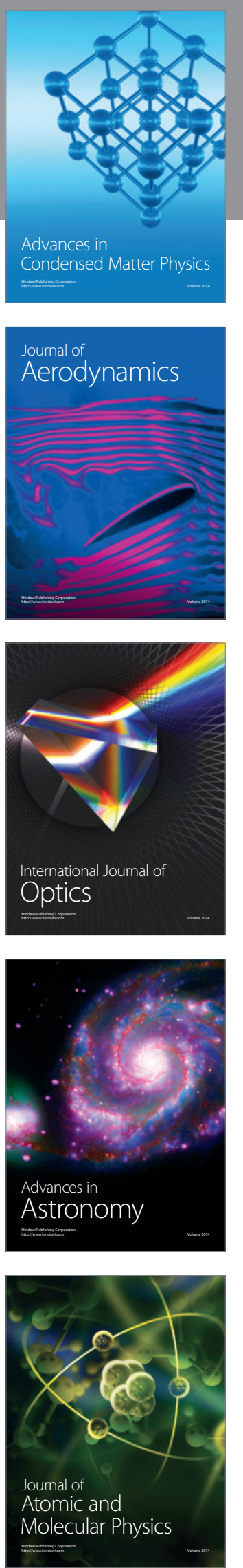